\title{
Network Analysis on Herbal Formulas from Wenrejingwei and Shang Han Lun
}

\author{
Anna $\mathrm{Kim}^{1}$, Sang-Hyun $\mathrm{Kim}^{1}$, Yong-Taek $\mathrm{Oh}^{2}$ * \\ ${ }^{1}$ KM Data Division, Korea Institute of Oriental Medicine, Daejeon, Republic of Korea \\ ${ }^{2}$ Department of Diagnostics, College of Korean Medicine, Woosuk University, Jeonju, Republic of Korea
}

Received June 25, 2021

Reviewed July 20, 2021

Accepted August 2, 2021

*Corresponding Author

Yong-Taek Oh

Department of Diagnostics, College of

Korean Medicine, Woosuk University, 61

Seonneomeo 3-gil, Wansan-gu, Jeonju

54986 , Republic of Korea

Tel: +82-63-290-9026

E-mail: ohyt@woosuk.ac.kr, ydydxor@gmail.com
Objectives: This study aims to describe the utilization of herbal formulas from Wenrejingwei by using network analysis and understand the treatment of acute exogenous febrile diseases.

Methods: We constructed a matrix of high-frequency herbal combinations (HCs) from Wenrejingwei and Shang Han Lun and cluster networks based on cohesive analysis. Network analysis was performed to compare the results.

Results: The results of the high-frequency HC network in Wenrejingwei showed cohesive patterns in three categories corresponding to dampness-heat and warm-fever treatment. Compared to the Shang Han Lun network, the Wenrejingwei network indicated a careful approach in the use of pungent and warm herbs such as Guizhitang. Moreover, the combination of Scutellaria baicalensis and Coptis chinensis along with the use of herbs strengthening yin, such as Ginseng Radix and Liriopes Radix, provide evidence of a holistic approach in the treatment of exogenous febrile diseases by considering the balance of the human body damaged by heat.

Conclusion: The results of this study could help select appropriate herbal formulas and treatment methods for treating Onbyeong and modern acute febrile infectious diseases.

Keywords: acute febrile diseases, herbal medicine, network analysis, wenrejingwei, wenbing

\section{INTRODUCTION}

Due to the recent outbreak of various infectious diseases such as the coronavirus disease 2019, Middle East respiratory syndrome, novel influenza, and severe acute respiratory disease, there is an increasing interest in Korean Medicine, specifically in Wenbing - the practice that focuses on acute febrile diseases. Wenbing focuses on externally contracted febrile diseases related to warm weather. These diseases have a pathogenic pattern similar to that of modern acute febrile diseases and can be divided into warm-heat and dampness-heat types.

Wenrejingwei is a representative publication of studies on Wenbing written by Wangshixiong (王士雄, 1808-1868), a prominent medical practitioner and scholar from the Chinese Qing Dynasty. The book has structurally organized medical theories and formulas from Neijing and earlier Wenbing studies [1].

Studies in Wenrejingwei are mostly qualitative analyses that focus on academic theories or provide an overview of the literary value of Wenrejingwei. This study aims to find the characteristics of key herbal constituents and analyze Wenrejingwei systematically by analyzing its formulas and their herbal combinations (HCs). As 30\% of the formulas from Wenrejingwei are cited from Shang Han Lun, the key herbal constituents of formulas from Wenrejingwei were compared to those from Shang Han Lun. Further, this study aimed to study the use of these herbal formulas in Wenrejingwei for Wenbing treatment in Korean medicine. 


\section{MATERIALS AND METHODS}

\section{Composition of herbal networks}

We selected 113 formulas from Wenrejingwei [2], 113 formulas from Shang Han Lun [3], and 36 formulas from Wenrejingwei cited in Shang Han Lun and processed them by determining the combination of each formula's constituent herbs and their frequencies. The following networks were constructed: Wenrejingwei network (W-Net), Shang Han Lun network (SNet), Wenrejingwei-Shang Han Lun network (WS-Net). The nodes in the network consisted of the constituent herbs and the links between the nodes were the relationships used in the HCs. The weight is the value of the link.

\section{Network analysis}

As the average frequencies of the nodes and their combinations, as well as minimum and maximum values, were different for each network, we selected the maximum $\mathrm{HC}$ weight value showing alteration in the tendency of the number of HCs for further analysis $[4,5]$.

In this study, three experts agreed that an alteration of over $30 \%$ in the number of HCs could be considered a valid alteration of tendency. Therefore, we selected the maximum HC weight that was equivalent for all three networks as the appropriate cut-off (Fig. 1). The cut-off weight values for W-Net, SNet, and WS-Net were 5, 6, and 4, respectively. Each network was subjected to cohesion analysis using Netminer ver.4.4.3.b. (Cyram Inc, Seoul, Korea).

\section{RESULTS AND DISCUSSION}

The results of the network analysis of $\mathrm{W}$-Net are shown in Fig. 2A. The key herb combinations of Wenrejingwei are G1, G2, and G3: Wulingsan (G1; Alisma canaliculatum, Poria cocos, Atractylodis Rhizoma Alba, Umbrella Polypore, Citri Reticulatae, Cinnamomi Cortex Spissus); G2 (Scutellaria baicalensis and Coptis chinensis); and Pingweisan (G3; Atractylodis Rhizoma, Citri Reticulatae, Magnoliae Cortex, Zingiberis Rhizoma Recens, Zizyphi Fructus). Both G1 and G3 formulas contain herbs that have a bland flavor and remove water, leading to the "spreading of dampness" in the spleen and stomach by diarrhea and urination. The herbs in G2 reduce heat and can be used for the treatment of the warm-heat type of Wenbing. The results of HCP network analysis show that the treatment of Wenbing in Wenrejingwei is based on the use of warm-heat and dampnessheat types of HCs.

The results of S-Net analysis are shown in Fig. 2B. The combination of Scutellaria baicalensis and Coptis chinensis is seen in both Wenrejingwei and Shang Han Lun networks. However, unlike the Wenrejingwei network, the Shang Han Lun network has HCs that are pungent and warm, such as Zingiberis Processum Rhizoma, whereas the Wenrejingwei network has HCs that strengthen yin, such as Ginseng Radix and Armeniacae Semen (Fig. 2A.G2). This can be better understood with the example of Gongbujianshi (攻補兼施), which is a treatment method that reduces heat and simultaneously strengthens the qi damaged by summer pathogens [6]. In other words, this approach aggressively treats local heat symptoms while simultaneously using a holistic approach to balance the human body that has been damaged by heat [2].

The results of WS-Net analysis are shown in Fig. 2C. WS-

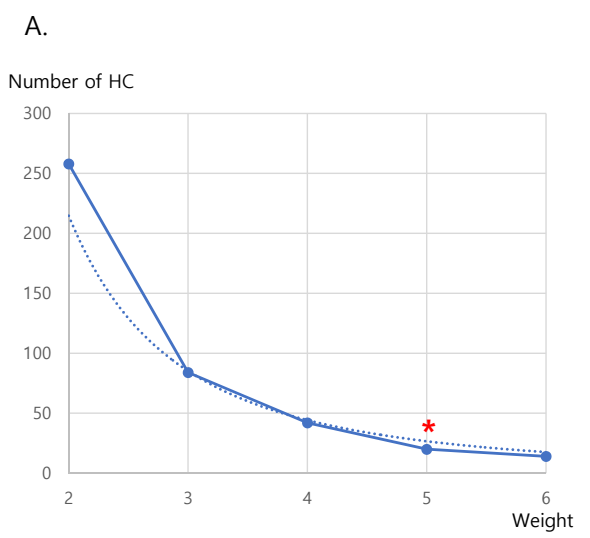

B.

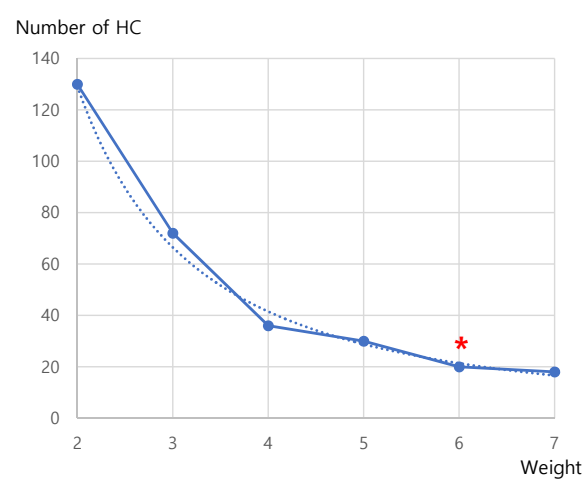

c.

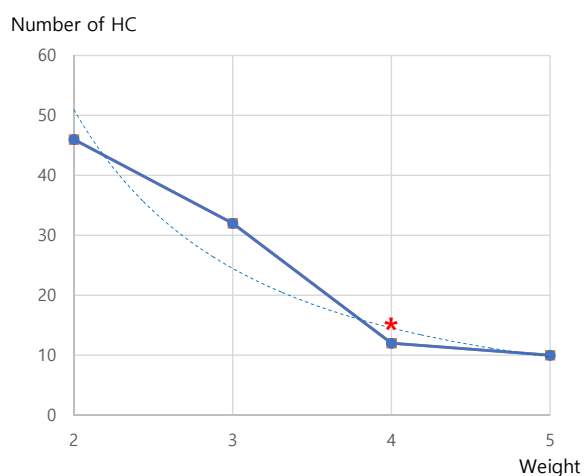

Figure 1. (A) Cut off weight value for W-net (value : 5). (B) Cut off weight value for S-net (value : 6). (C) Cut off weight value for WS-net (value : 4). 
A.
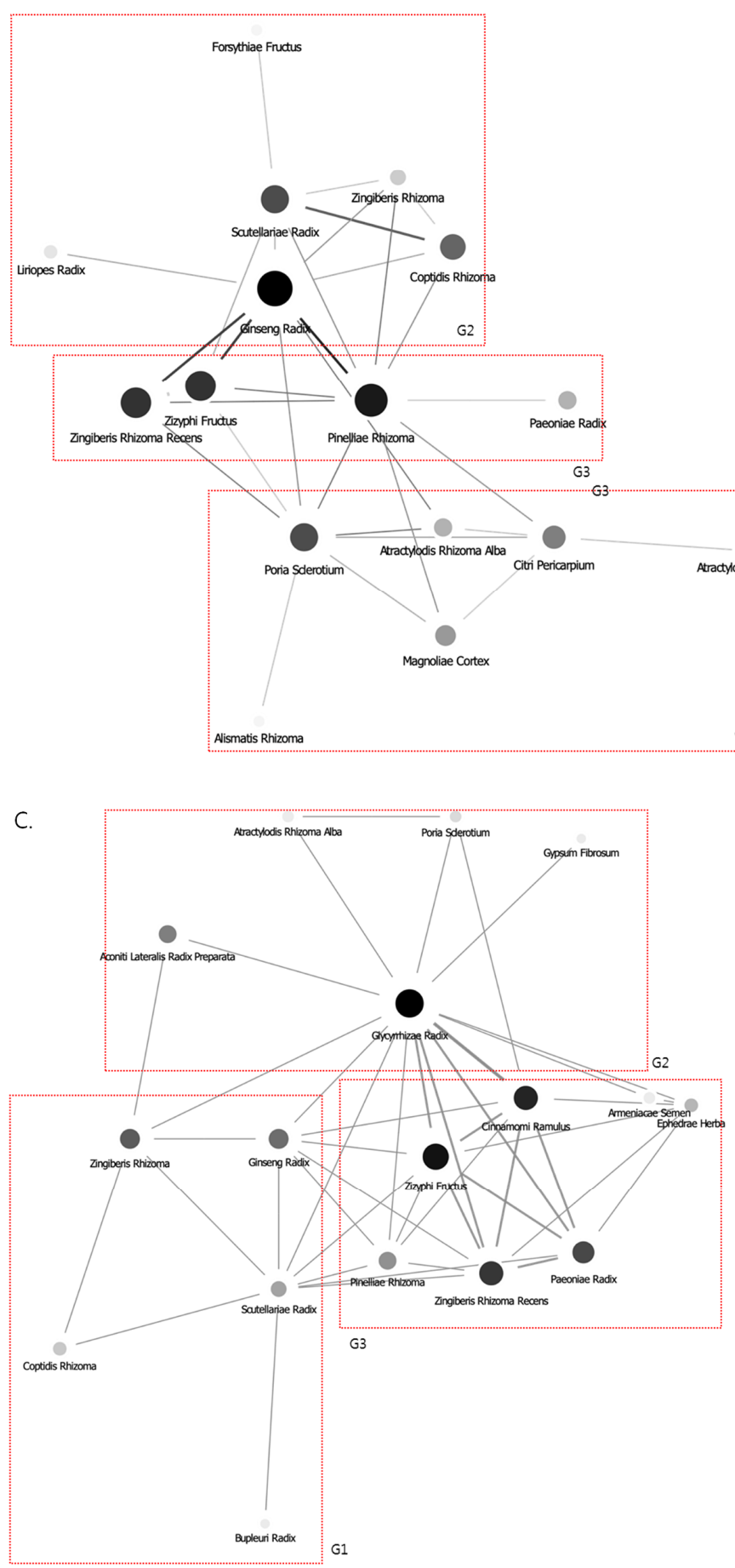

Net contains formulas that are cited from Shang Han Lun, which contains HCs from Guizhitang (Fig. 2B.G3) (Cinnamomi ramulus, Paeonia japonica, Zingiberis Rhizoma Recens, Zizyphi Fructus, Glycyrrhizae Radix) that are pungent and warm. However, WS-Net does not contain pungent and warm herb combinations found in Guizhitang. This shows the careful ap-
B.

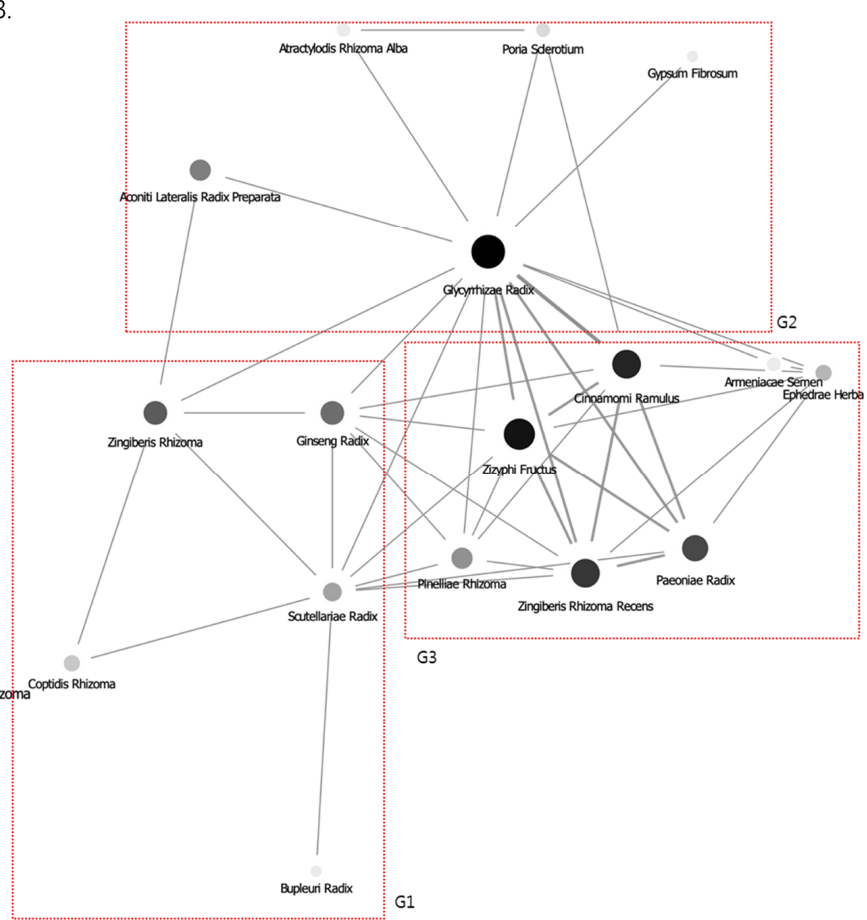

Figure 2. (A) Network analysis for herbs of Wenrejingwei formulas (Modularity analysis. Cut off weight value : 5). (B) Network analysis for herbs of Shanghanlun formulas (Modularity analysis. Cut off weight value : 6). (C) Network analysis for herbs of Shanghanlun formulas from Wenrejingwei (Modularity analysis. Cut off weight value : 4).

proach in Wenbing treatment toward the use of pungent and warm herbs. In addition, the results of these network analyses describe the methodology of Wangshixiong, the writer of Wenrejingwei, in using specific formulas from Shang Han Lun to treat Wenbing while excluding formulas deemed inappropriate for Wenbing treatment. 
This study focused on the relationship between herbs and analyzed the cohesive pattern of $\mathrm{HC}$ networks frequently used in Wenrejingwei. The limitations of this study are that it did not explore the activity of these herbs and only included representative publications instead of all publications covering Wenbing.

The results show the importance in HCs in Wenrejingwei, such as Wuling San and Ping Wei San, for the dampness-heat type of Wenbing, and Scutellaria baicalensis and Coptis chinensis for the warm-heat type of Wenbing. Unlike Shang Han Lun, Wenrejingwei approaches the reduction of heat by using Ginseng Radix and Armeniacae Semen and simultaneously preserving body fluids. This study analyzed the structural characteristics of formulas from Wenrejingwei and the mode of application along with the treatment objective in Korean medicine.

\section{CONCLUSION}

These results show that key HCs or herbal constituents can be considered for the treatment of Wenbing in addition to formulas traditionally used in clinical practice. Our study also provides a new perspective of using a holistic approach of reducing heat and strengthening yin when treating modern acute exogenous febrile diseases.

\section{SUPPLEMENTARY MATERIALS}

Supplementary data is available at https://doi.org/10.3831/ KPI.2021.24.3.138.

\section{ACKNOWLEDGEMENT}

This work was supported by a National Research Founda- tion (NRF) grant funded by the Korea government (MSIP) (No. 2017R1C1B5018236) and the Korea Institute of Oriental Medicine (grant number KSN2013110).

\section{CONFLICT OF INTEREST}

The authors declare no conflict of interest.

\section{ORCID}

Anna Kim, https://orcid.org/0000-0002-4607-1328

Sang-Hyun Kim, https://orcid.org/0000-0002-8512-8999

Yong-Taek Oh, https://orcid.org/0000-0001-8973-0795

\section{REFERENCES}

1. Feng GK. [Analysis on the academic thoughts of warm diseases in 《Wenrejingwei》]. Liaoning J Tradit Chin Med. 2011;38(12): 2357-8. Chinese.

2. Wang SX. Pingzhu Wenrejingwei. Seoul: Jipmoondang; 2013. 6-7 p.

3. Chae IS. Shanghanlun yeokjeon. Seoul: Publisher of Komoon; 1987.

4. Kim A, Kim HJ, Song BY, Lee SH, Cho YH, Oh YT. Network analysis on herbal combinations in Korean medicine for insomnia. J Korean Med Class. 2018;31(4):69-78.

5. Oh YT, Kim HJ, Kim A. Study of structural analysis on formulas from ${ }^{\circledR}$ Onbyungjobyun $₫$ using network analysis. Herb Formula Sci. 2019;27(1):65-71.

6. Liu JY. Liujingyuan's lecture on febrile disease. Paju: Fishwood; 2017. 